\title{
Prediction of pathologic stage in non-small cell lung cancer using machine learning algorithm based on CT image feature analysis
}

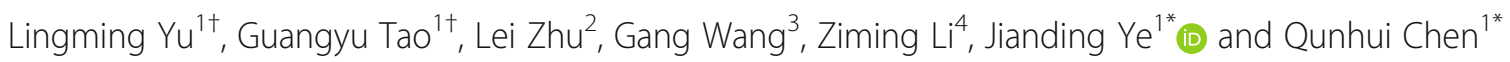

\begin{abstract}
Purpose: To explore imaging biomarkers that can be used for diagnosis and prediction of pathologic stage in non-small cell lung cancer (NSCLC) using multiple machine learning algorithms based on CT image feature analysis.

Methods: Patients with stage IA to IV NSCLC were included, and the whole dataset was divided into training and testing sets and an external validation set. To tackle imbalanced datasets in NSCLC, we generated a new dataset and achieved equilibrium of class distribution by using SMOTE algorithm. The datasets were randomly split up into a training/testing set. We calculated the importance value of $C T$ image features by means of mean decrease gini impurity generated by random forest algorithm and selected optimal features according to feature importance (mean decrease gini impurity $>0.005$ ). The performance of prediction model in training and testing sets were evaluated from the perspectives of classification accuracy, average precision (AP) score and precision-recall curve. The predictive accuracy of the model was externally validated using lung adenocarcinoma (LUAD) and lung squamous cell carcinoma (LUSC) samples from TCGA database.

Results: The prediction model that incorporated nine image features exhibited a high classification accuracy, precision and recall scores in the training and testing sets. In the external validation, the predictive accuracy of the model in LUAD outperformed that in LUSC.

Conclusions: The pathologic stage of patients with NSCLC can be accurately predicted based on CT image features, especially for LUAD. Our findings extend the application of machine learning algorithms in CT image feature prediction for pathologic staging and identify potential imaging biomarkers that can be used for diagnosis of pathologic stage in NSCLC patients.
\end{abstract}

Keywords: Non-small cell lung cancer (NSCLC), Computed tomography (CT), Radiomics, Machine learning algorithm

\footnotetext{
*Correspondence: yejiandingchest@sohu.com; chenqunhui312@163.com

${ }^{\dagger}$ Lingming Yu and Guangyu Tao contributed equally to this work.

'Department of Radiology, Shanghai Chest Hospital, The Affiliated Chest

Hospital of Shanghai Jiaotong University, No. 241 Huaihai West Road, Xuhui

District, Shanghai 200030, China

Full list of author information is available at the end of the article
}

(C) The Author(s). 2019 Open Access This article is distributed under the terms of the Creative Commons Attribution 4.0 International License (http://creativecommons.org/licenses/by/4.0/), which permits unrestricted use, distribution, and reproduction in any medium, provided you give appropriate credit to the original author(s) and the source, provide a link to the Creative Commons license, and indicate if changes were made. The Creative Commons Public Domain Dedication waiver (http://creativecommons.org/publicdomain/zero/1.0/) applies to the data made available in this article, unless otherwise stated. 


\section{Background}

Lung cancer is one of the most frequent types of malignancy and a leading cause of cancer-associated mortality worldwide [1]. In clinical management, lung cancer can be classified in two main categories, non-small cell lung cancer (NSCLC) and small cell lung cancer, with the former occupying approximately $85 \%$ of lung cancers [2, 3]. NSCLC represents a heterogeneous group of cancers mainly composed of lung squamous cell carcinoma (LUSC) and lung adenocarcinoma (LUAD) [4, 5]. However, the 5-year survival of NSCLC remains dismal, and $70 \%$ cases are diagnosed after the onset of advanced local or metastatic disease. The prognosis varies widely according to tumor staging at diagnosis [6]. Unfortunately, merely $15 \%$ of cases are not diagnosed until late stage [7], and thus the accurate prediction of pathologic stage for patients with lung cancer is of utmost importance.

Staging plays a crucial role in the evaluation of a patient as it defines the actual extent of the disease. Pathologic tumor stage is considered a pivotal factor relating to survival in NSCLC, and the 5-year survival rates vary from $83 \%$ in pathological stage IA to $23 \%$ in stage IIIA tumors [8]. Accurate staging is conducive to developing the effective medical treatment and to predicting patient prognosis. With the widespread application and advanced imaging technology in screening and diagnosis, the pathologic stages of more tumors have been diagnosed. However, the rate of recurrence remains unsatisfactory and ranges between 15 and 30\%, even after complete surgical resection [9]. Pathologic staging of LUSC and LUAD remains a challenge for the physician using individual pretreatment variables.

Recent advances in radiography, such as high-resolution computed tomography and the widespread practice of low-dose helical computed tomography (CT) for screening of tumors, have led to an increase in the early detection of NSCLC [10]. CT has been widely used as a noninvasive diagnostic modality for diagnosis, clinical staging, survival prediction, surveillance of therapeutic response in patients with NSCLC [11-13]. Tumor phenotypic differences, such as irregular shapes and heterogeneity, can be measured using radiomic features derived from $\mathrm{CT}$ images.

Radiomics is an emerging technique that utilizes high-throughput quantitative image features for diagnosis and prognosis [14]. Radiomics focuses on systematic quantification of the tumor phenotype by effectively extracting and analyzing massive image data $[15,16]$. An increasing number of studies have suggested that CT image features have high diagnostic and predictive values in clinical pathologic staging of diseases and clinical outcomes [17-19]. For instance, in a previous research of Kim et al., CT radiomics features, including roundness and grey-level nonuniformity, were verified as predictive biomarkers of survival in LUAD [20]. Ravanelli et al. performed a texture analysis on contrast-enhanced computed tomography images in advanced LUAD and uncovered an independent predictive indicator for treatment response [21]. Nonetheless, few image features can be used for accurate prediction of pathologic stage in patients with NSCLC.

Herein, our study applied a series of machine learning algorithm, and explored potential imaging biomarkers that can be used for diagnosis and prediction of pathologic stage in NSCLC based on a CT image feature analysis.

\section{Methods}

Data sets

A total of 145 patients with pathologically confirmed stage IA to IV NSCLC were included in this study. The patient cohort was comprised of three datasets, including NSCLC $(n=87)$, LUAD $(n=24)$ and LUSC $(n=34)$. NSCLC samples were averagely divided into a training set and a testing set, while LUAD and LUSC data sets were used for external validation. CT images of all patients were publicly available on the cancer imaging archive (TCIA). The clinico-pathologic characteristics of patients in NSCLC, LUAD, and LUSC cohorts were shown in Table 1 . The inclusion criteria were those who were newly diagnosed or treatment-naive NSCLC and pathologically confirmed stage IA to IV lung adenocarcinoma and squamous cell carcinoma, as well as had pre-treatment $\mathrm{CT}$ images. The exclusion criteria are the patients who were treated with surgery or chemoradiation therapy and contained incorrect staging information. The TRIPOD checklist is appended as Additional file 1: Table S1.

Table 1 Patient and tumor characteristics in the training and validation sets

\begin{tabular}{lllll}
\hline & $\begin{array}{l}\text { NSCLC } \\
(N=87)\end{array}$ & $\begin{array}{l}\text { TCGA-LUAD } \\
(N=24)\end{array}$ & $\begin{array}{l}\text { TCGA-LUSC } \\
(N=34)\end{array}$ & $P$ value \\
\hline sex & & & 19 & 0.033 \\
male & 58 & 9 & 15 & \\
female & 29 & 15 & & 0.513 \\
Overall stage & & & 3 & \\
IA & 14 & 5 & 10 & \\
IB & 28 & 5 & 5 & \\
IIA & 5 & 2 & 9 & \\
IIB & 22 & 3 & 4 & \\
IIIA & 10 & 7 & 2 & \\
IIIB & 3 & 1 & 1 & \\
IV & 5 & 1 & & \\
\hline
\end{tabular}




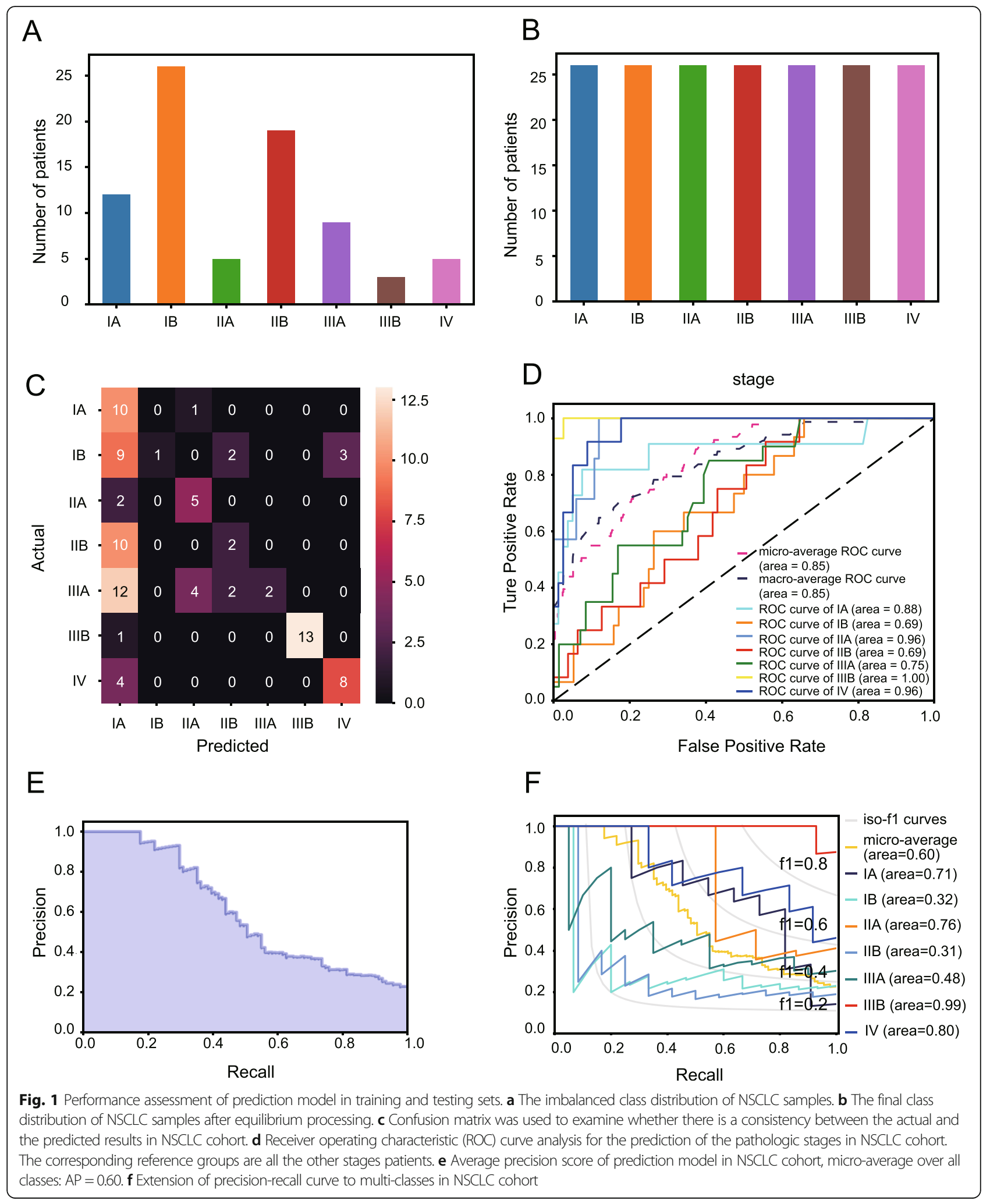




\section{Lesion recognition and region-of-interest segmentation} by 3D-slicer software

It is well known that manual segmentation is required before extracting radiological features. All patient images were loaded and processed in the original DICOM format. We used 3D-Slicer software (https://www.slicer.org/) to load CT image files and RTSTRUCT files for mapping sub-regions of lesions. Apply the segment editor module to change the main representation from a flat outline to a binary label map. The 3D image file and the binary mask tag file are saved by the 3D-Slicer as NRRD format files for the next feature extraction step.

\section{Extracting features from CT images using Pyradiomics}

By segmenting the region of interest of the tumor, features can be extracted and four types of imaging features (shape, intensity, texture, and wavelet) can be identified. We used pyradiomics (http://readthedocs. org/projects/pyradiomics/) which is an open source python package to perform feature extraction tasks. Some quantitative features are as follows: first-order features, shape features, gray level co-occurrence matrix (GLCM) features. In addition to the shape features, other features can be measured on the original or derived image, while the shape descriptor is independent of the gray value and is extracted from the label mask (http://pyradiomics.readthedocs.io/en/latest/ Features.html).

\section{Data preprocessing}

First, we should confirm whether the original class distribution of NSCLC cohort was balanced. If not, over sampling would be performed by means of SMOTE algorithm, to tackle the curse of imbalanced datasets in machine learning and to achieve equilibrium of class distribution by producing a new data set. The newly-generated data sets were then split up into a training set and a testing set.

\section{Predictive modeling and feature selection}

Considering some redundant and irrelevant features that may influence classification accuracy of the prediction model, we calculated the importance value of CT image features by means of Random Forest algorithm, and then selected optimal features in accordance with feature importance (mean decrease gini impurity >0.005) for modeling. Random forest is a tree-based ensemble learning method for regression and classification, developed by Leo Breiman [22]. It is widely applied in medicine, and has proven to be an easy-to-use and highly accurate predictive method [23]. From the methodological perspective of feature selection, random forest is a kind of embedded feature selector which can automatically produce the relative importance of features during the model training process. Here, the classification accuracy of the random forest was evaluated using out of bag (OOB) error, which is an unbiased estimate of random forest generalization error. We used the python module scikit-learn to perform all the above modelling process using default parameters. In view of the limited sample size of each stage, we also performed all the aforementioned analysis on binarized stage of early (stage I/II) and late (stage III/IV).

\section{Classification accuracy of prediction model}

To evaluate the performance of prediction model in training and testing sets, receiver operating characteristics (ROC) curves were plotted to display classification performance in the testing set and the external validation set. The ROC curve is a comprehensive index that reflects false positive rate and true positive rate of continuous

Table 2 Feature importance

\begin{tabular}{ll}
\hline Feature & Importance \\
\hline wavelet-HHH_firstorder_RootMeanSquared & 0.007136 \\
log-sigma-2-0-mm-3D_firstorder_RootMeanSquared & 0.006829 \\
wavelet-HHL_glcm_InverseVariance & 0.006782 \\
wavelet-HHL_glcm_Idn & 0.006155 \\
wavelet-HHL_firstorder_Variance & 0.005531 \\
wavelet-HHL_glszm_SmallAreaHighGrayLevelEmphasis & 0.00533 \\
wavelet-HHL_glcm_InverseVariance & 0.005291 \\
wavelet-HHL_glcm_Imc1 & 0.005072 \\
wavelet-HHL_glrlm_LongRunLowGrayLevelEmphasis & 0.005063 \\
wavelet-HHL_glcm_Idmn & 0.004948 \\
wavelet-HHL_glrlm_GrayLevelVariance & 0.004713 \\
wavelet-HHL_glszm_LargeAreaLowGrayLevelEmphasis & 0.004235 \\
wavelet-HHL_glcm_Idm & 0.004189 \\
wavelet-HHL_glrlm_ShortRunHighGrayLevelEmphasis & 0.004122 \\
wavelet-LLL_glrlm_LongRunHighGrayLevelEmphasis & 0.003965 \\
wavelet-HLH_glcm_JointEnergy & 0.003955 \\
wavelet-HHL_gldm_LargeDependenceEmphasis & 0.003925 \\
original_glszm_ZoneVariance & 0.003886 \\
log-sigma-2-0-mm-3D_glcm_ClusterProminence & 0.003725 \\
wavelet-HHL_firstorder_Median & 0.003717 \\
wavelet- & 0.003683 \\
HHL_gldm_SmallDependenceHighGrayLevelEmphasis & \\
wavelet-HHL_glrlm_LongRunHighGrayLevelEmphasis & 0.003615 \\
wavelet-HHL_glcm_DifferenceVariance & 0.003579 \\
log-sigma-4-0-mm-3D_glszm_GrayLevelNonUniformity & 0.003525 \\
wavelet-LLH_firstorder_RootMeanSquared & 0.003449 \\
wavelet-LLL_glszm_SizeZoneNonUniformityNormalized & 0.003391 \\
wavelet-HLL_glszm_GrayLevelVariance & 0.003327 \\
log-sigma-4-0-mm-3D_glrlm_ShortRunEmphasis & 0.003289 \\
\hline
\end{tabular}


A

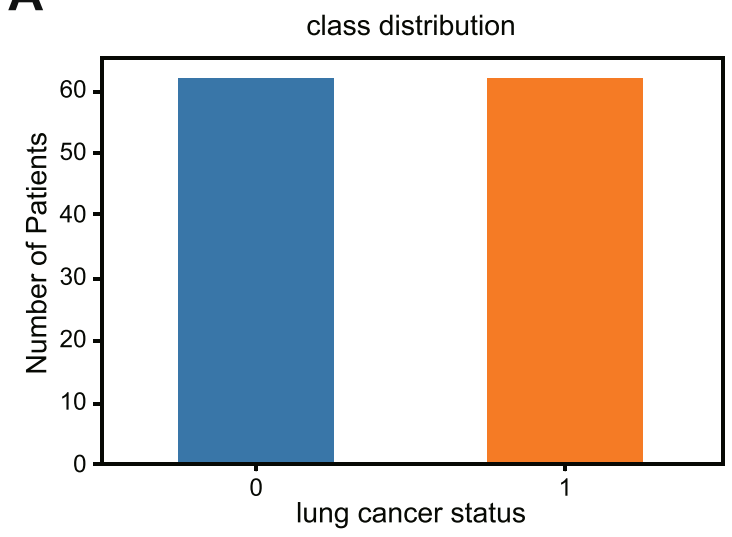

C

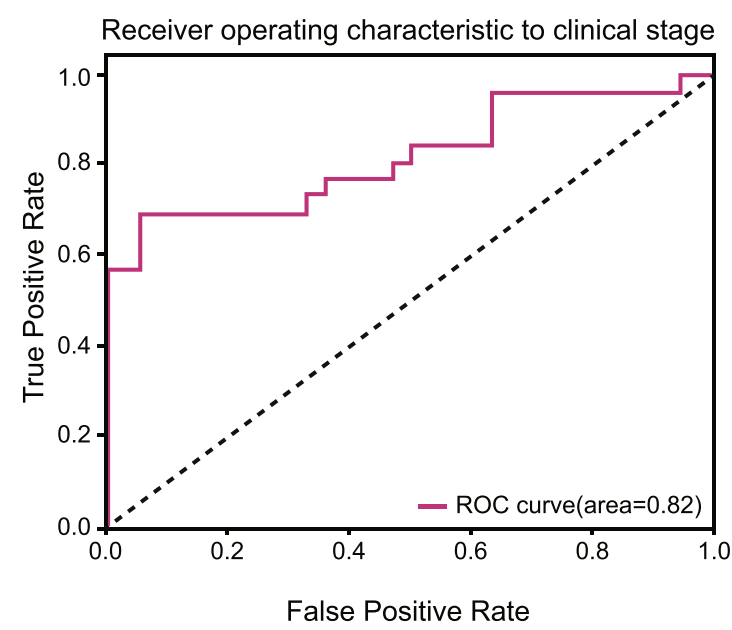

E

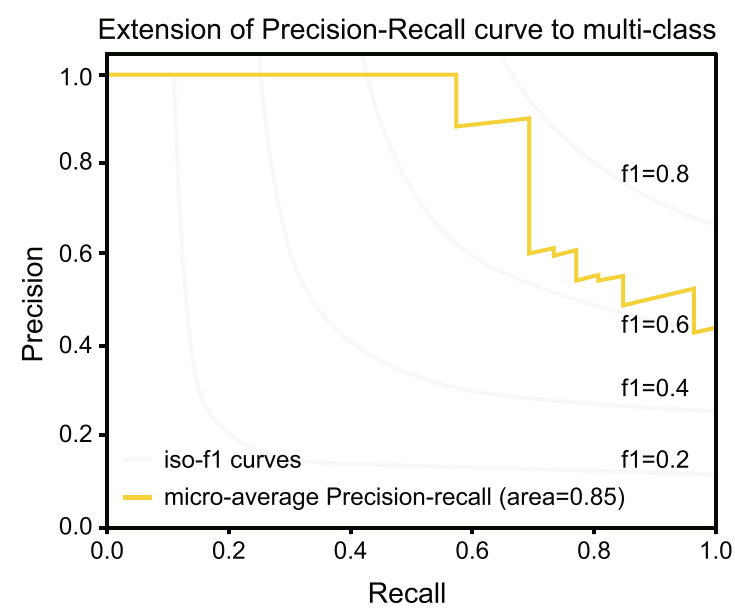

B

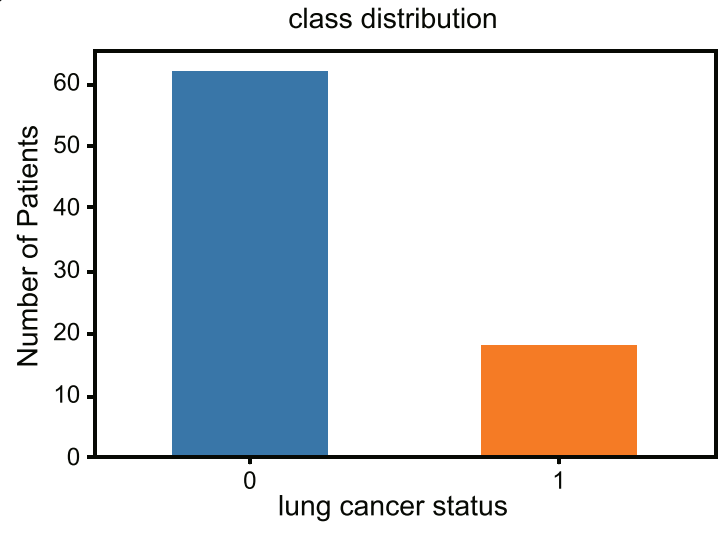

D

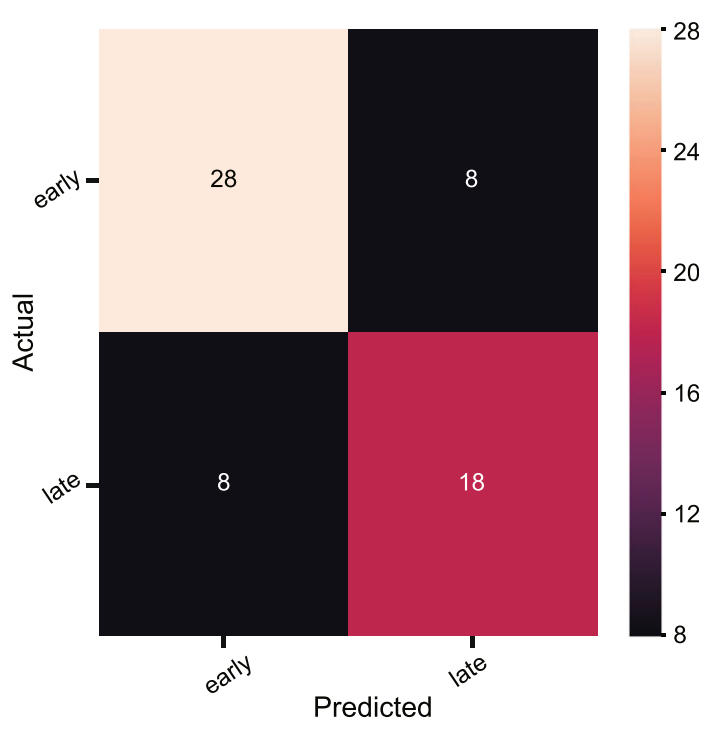

F

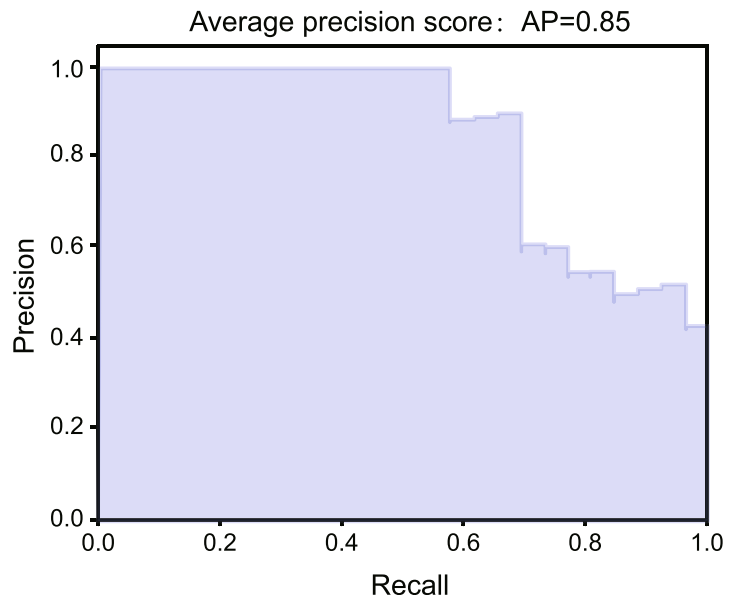

Fig. 2 (See legend on next page.) 
(See figure on previous page.)

Fig. 2 Performance assessment of prediction model in training/testing sets in binarized predictive scenario. a The imbalanced class distribution of NSCLC samples. $\mathbf{b}$ The final class distribution of NSCLC samples after equilibrium processing. $\mathbf{c}$ Receiver operating characteristic (ROC) curve analysis for the prediction of the pathologic stages in NSCLC cohort. $\mathbf{d}$. Confusion matrix was used to examine whether there is a consistency between the actual and the predicted results in NSCLC cohort. e Precision-recall curve in NSCLC cohort. $\mathbf{f}$ Average precision score of prediction model in NSCLC cohort

variables. The area under the curve (AUC) was an evaluation measure for model performance.

In addition, confusion matrix was applied to examine whether there is a consistency between the predicted and actual results. Confusion matrix is a useful tool to evaluate the performance of classifiers in their ability to classify multi-classed objects in addition to ROC curves. In this study, we focused on the generalization properties of learning algorithm for multiclass classification problems and used the confusion matrix of a classifier as a measure of its quality. We also used accuracy score, the ration of number of correctly classified samples to the number of all the samples, to evaluate the model predictive performance. Finally, we computed a new model by using original features, the accuracy score of which could be calculated according to the chosen optimal features.

\section{Assessment of prediction model using precision-recall curves}

In addition to evaluating accuracy of prediction model using ROC curves, Precision-Recall metric was also employed to estimate the output quality of the classifier. Precision-Recall curves is more informative when evaluating binary classifier on imbalanced datasets with performance measures such as precision and recall metrics. A high area under the curve of a precision-recall curve can be detected with either high precision or high recall, which also suggests a low false positive rate or a low false negative rate. High scores for both show that the classifier is returning accurate results (high precision), as well as returning a majority of all positive results (high recall). Moreover, the higher f1-score, the more stable the classification model.

Considering the limitation of single metrics-precision, recall and f1-score, we decided to adopt average precision score and precision-recall to each class to assess the overall capacity. Here, average precision (AP) is used to measure the accuracy of the classifier using weighted mean of precisions achieved at each threshold. Furthermore, the output would be binarized if the precision-recall curve and average precision were extended to multi-class or multi-label classification. The precision-recall curve can be plotted through considering each element of the label indicator matrix, which is considered a binary prediction (micro-averaging).

\section{Results}

Clinico-pathologic characteristics

A total of 145 were pathologically diagnosed with NSCLC, including LUSC, LUAD, or other subtypes of NSCLC. In the training/testing sets with 87 NSCLC patients, there were 58 male patients and 29 female ones. Furthermore, the LUAD cohort comprised of 24 patients and the LUSC cohort consisting of 34 ones were separately used for the external validation of the model. As shown in Table 1, no discrepancy was detected between the training/testing sets and the validation set in gender $(P=0.14)$, while there was a significant difference found between three cohorts in terms of clinico-pathologic stages $(P=0.02)$. Clinical information for NSCLC patients from TICA database was provided in Additional file 2: Table S2.

\section{Identification of imaging biomarkers}

Through observation on data of each group, we confirmed the imbalanced class distribution of original NSCLC samples in the training set (Fig. 1a), and then conducted over sampling by using random oversampling. Another machine learning algorithm, SMOTE, was subsequently employed to generate a new balanced data set for the following analyses (Fig. 1b).

Given that some redundant or irrelevant features in the new data set may exert an influence on the classifying effects of the model, the importance value of CT image features was first calculated by means of Random Forest algorithm (Table 2), followed by selection of the optimal features based on each feature importance (mean decrease gini impurity $>0.005$ ). In total, nine image features were chosen for modeling. These features are wavelet-HHH_firstorder_RootMeanSquared, log-sig ma-2-0-mm-3D_firstorder_RootMeanSquared, waveletHHL_glcm_InverseVariance, wavelet-HHL_glcm_Idn, wavelet-HHL_firstorder_Variance, wavelet-HHL_glszm_ SmallAreaHighGrayLevelEmphasis, wavelet-HHL_glcm InverseVariance, wavelet-HHL_glcm_Imc1, waveletHHL_glrlm_LongRunLowGrayLevelEmphasis, respectively. All the features are numerical features. There nine features can be utilized as the stage-predictive image biomarkers. Several features are easy to interpret. Root 


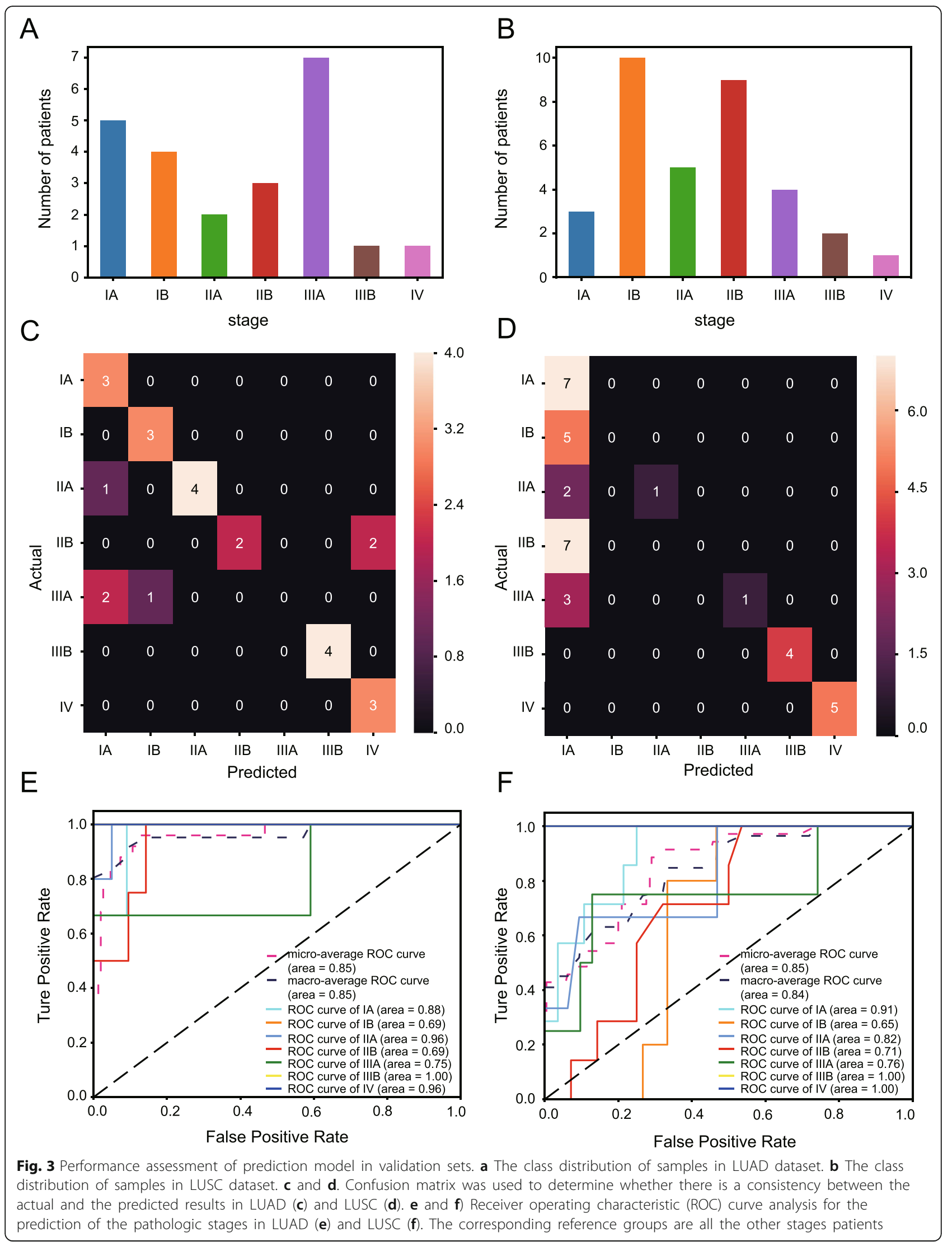




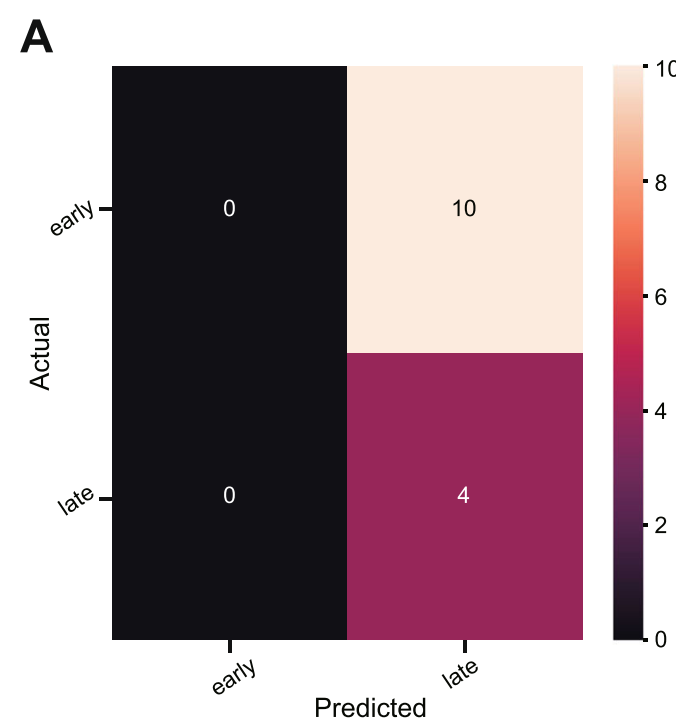

B
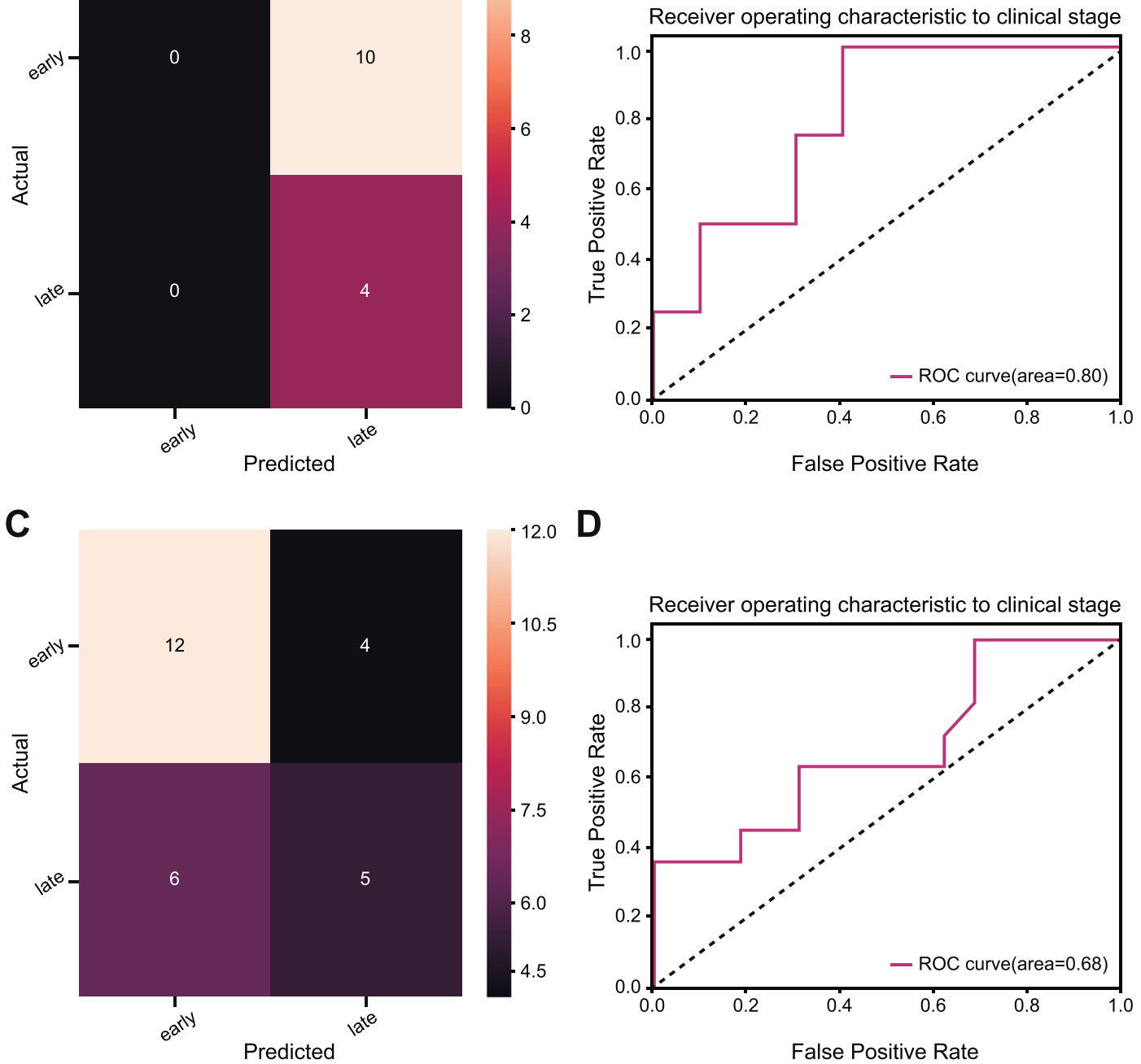

D

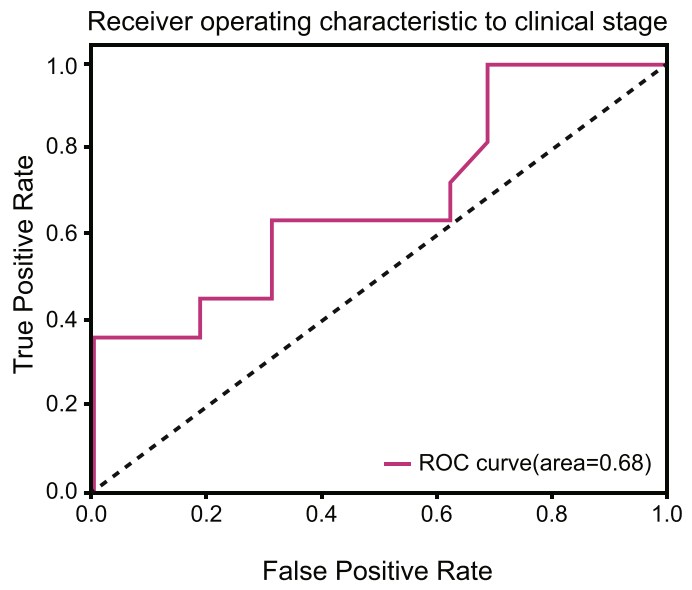

E

F
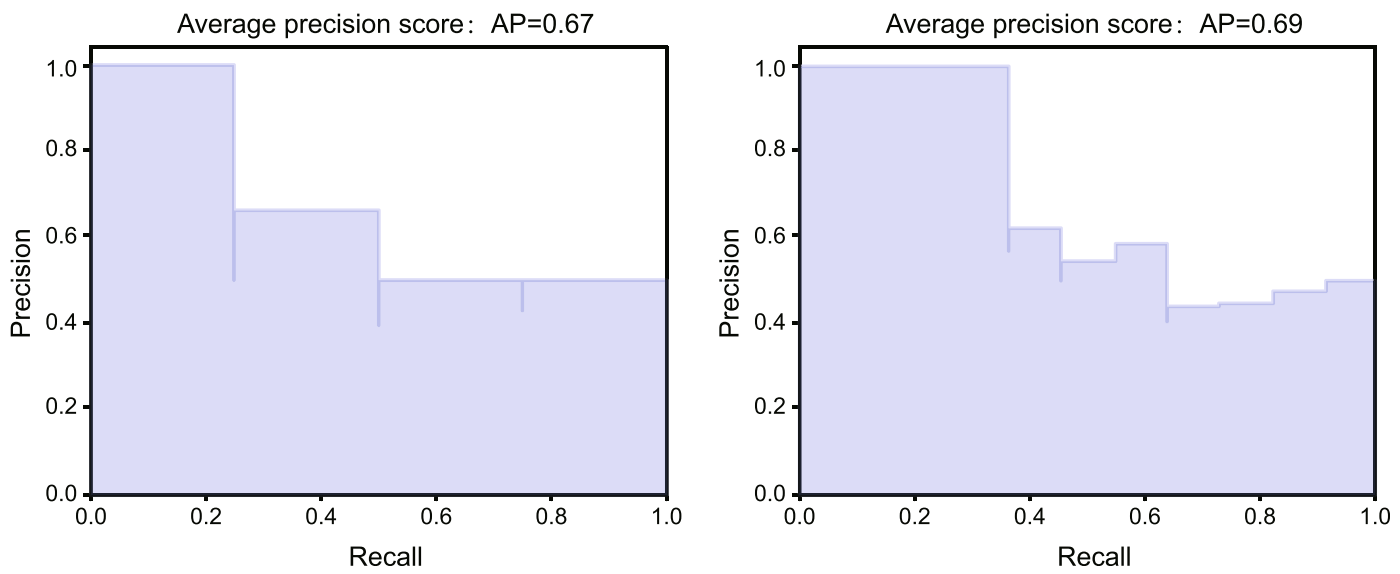

Fig. 4 (See legend on next page.) 
(See figure on previous page.)

Fig. 4 Performance assessment of prediction model in validation sets. in binarized predictive scenario. a \& c Confusion matrix was used to determine whether there is a consistency between the actual and the predicted results in LUAD (up) and LUSC (down). $\mathbf{b}$ \& $\mathbf{d}$ Receiver operating characteristic (ROC) curve analysis for the prediction of the pathologic stages in LUAD (up) and LUSC (down). e Average precision score of prediction model in LUAD. $\mathbf{f}$ Average precision score of prediction model in LUSC

mean squared (RMS) is the square-root of the mean of all the squared intensity values. It is a measure of the magnitude of the image values. As for inverse difference normalized (IDN), it is a measure of the local homogeneity of an image. Small Area High Gray Level Emphasis (SAHGLE) measures the proportion in the image of the joint distribution of smaller size zones with higher gray-level values. Long Run Low Gray Level Emphasis (LRLGLE) measures the joint distribution of long run lengths with lower gray-level values. Ultimately, the classification accuracy of the models was evaluated using OOB error, with 0.81 of original random forest model and 0.86 of limited feature model.

\section{Performance evaluation of prediction model in training sets}

The prediction model's performance was first assessed in the testing sets with equilibrium of class distribution and balanced data. In terms of classification accuracy, confusion matrix results confirmed that there was a consistency between the predicted and actual results, which suggested a better performance of the model in the classification of multi-class objects (Fig. 1c). Furthermore, ROC curve analysis also verified that the model could predict and distinguish pathologic stages of NSCLC with high accuracy of $0.69 \sim 1.00$ (Fig. 1d). Here, the accuracy scores of the original random forest model and limited feature model were 0.53 and 0.57 , respectively.

In addition to accuracy score, the prediction model was also evaluated from the perspectives of average precision score and precision-recall to each class. As exhibited in precision-recall curves (Fig. 1e, f), our prediction model not only yielded a higher average precision score (AP) of 0.60 (Fig. 1e), but achieved a better diagnostic performance for pathologic stages of NSCLC in terms of the extension of precision-recall curve to multi-classes (Fig. 1f). Corresponding results of binarized stage scenario is depicted in Fig. 2.

\section{Performance evaluation of prediction model in external validation sets}

In addition to the internal testing above, we also performed an external validation for the performance of the prediction model by using LUAD and LUSC data sets without preprocessing or equilibrium of class distribution. The original class distribution of samples in the LUAD cohort (Fig. 3a) and LUSC cohort (Fig. 3b) was displayed in Fig. 4. The machine learning algorithms in the external validation were same as those used in testing sets. In terms of the classifier performance in the LUAD data set, confusion matrix and ROC curves both indicated a high classification accuracy of the model, with AUC value of $0.69 \sim 1.00$ (Fig. 3c, d). Likewise, we also re-confirmed the consistency between the predicted and actual results in the LUSC data set (Fig. 3e). ROC curves also revealed that the model could distinguish the pathologic stages of LUSC with high accuracy of at least 65\% (Fig. 3f).

For average precision score and precision-recall to each class of the model, our precision-recall curves in the external validation set presented that the prediction model not only achieved higher average precision scores in both LUAD (AP = 0.84) and LUSC $(\mathrm{AP}=0.62)($ Fig. 5a, c) but yielded a better differentiation performance for pathologic stages of LUAD and LUSC in terms of the extension of precision-recall curve to multi-classes (Fig. 5b, d). Taken together, our prediction model that incorporated nine image features could predict and differentiate the pathologic stages of NSCLC accurately, and the predictive accuracy of the model in LUAD outperformed that in LUSC. Corresponding results of binarized stage scenario is depicted in Fig. 4. In addition, the association between Radiomic Score and TNM factors was separately evaluated as shown in Additional file 3: Table S3, and similar results were found in the NSCLC dataset.

\section{Discussion}

The present study revealed that image features extracted from CT scans was correlated with pathologic stage of patients with NSCLC. Our study further explored the function and application of machine learning in CT image feature analysis for pathologic staging, meanwhile, unveiled potential imaging biomarkers that can be used for diagnosis and prediction of pathologic stage in NSCLC. Ultimately, our prediction model that incorporated nine optimal characteristics was validated to be significantly effective in the prediction of lung cancer subtypes and pathologic tumor stages of LUAD and LUSC.

In recent years, radiomics plays an emerging role in cancer research, imaging biomarkers and clinical management. An increasing number of image characteristics have been reported to have high predictive and diagnostic values in NSCLC [12, 24]. For instance, Coroller et al. corroborated that $\mathrm{CT}$-based radiomic signature could predict distant metastasis in lung adenocarcinoma [25]. 

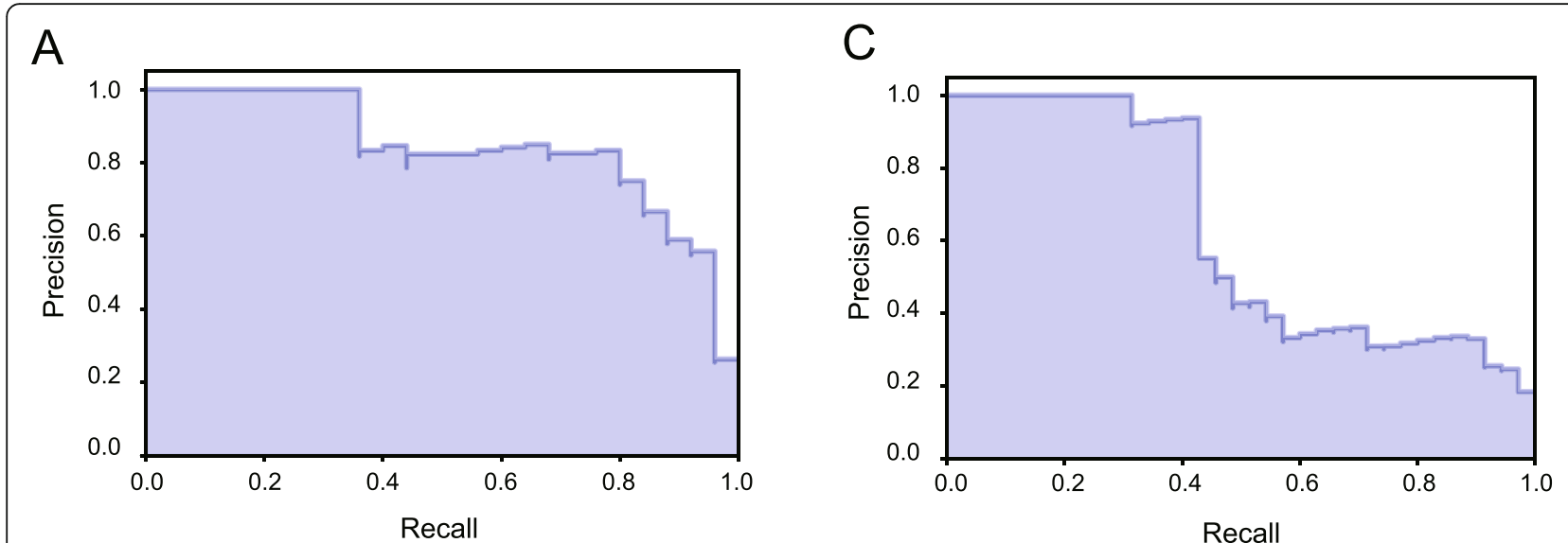

B

D
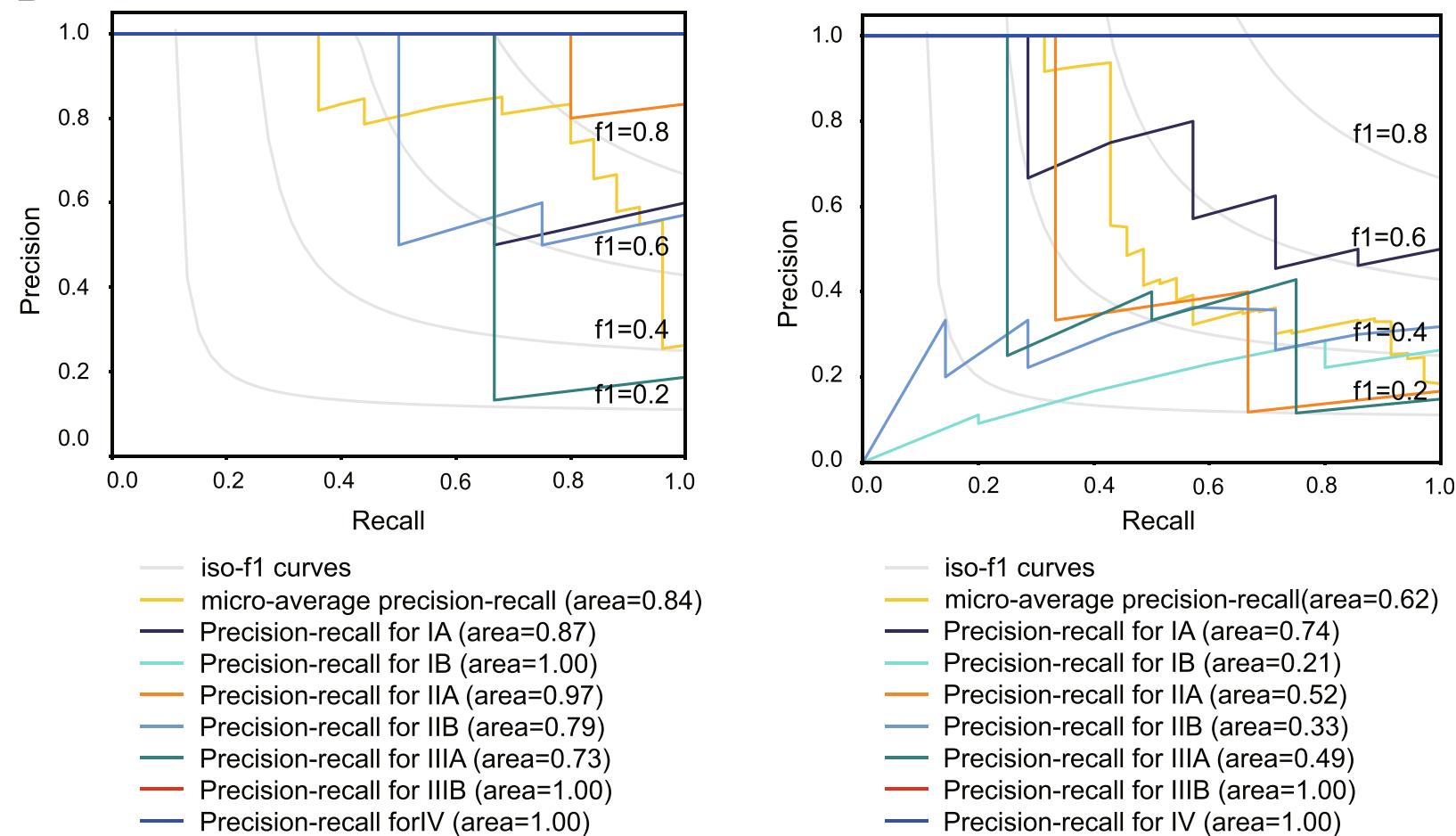

- iso-f1 curves

micro-average precision-recall (area $=0.84$ )

- Precision-recall for IA (area $=0.87$ )

- Precision-recall for IB (area=1.00)

- Precision-recall for IIA (area $=0.97$ )

- Precision-recall for IIB (area $=0.79)$

- Precision-recall for IIIA (area $=0.73$ )

- Precision-recall for IIIB (area $=1.00)$

- Precision-recall forlV (area $=1.00$ )

Fig. 5 Performance assessment of prediction model in validation sets in terms of precision and recall score. a Average precision score of prediction model in LUAD, micro-average over all classes: $A P=0.84$. $\mathbf{b}$ Extension of precision-recall curve to multi-classes in LUAD. The corresponding reference groups are all the other stages patients. c Average precision score of prediction model in LUSC, micro-average over all classes: $A P=0.62$. $\mathbf{d}$ Extension of precision-recall curve to multi-classes in LUSC. The corresponding reference groups are all the other stages patients

Ko et al. unraveled the predictive value of 18F-FDG PET and CT morphologic features for recurrence in pathological stage IA non-small cell lung cancer [26]. Chen et al. demonstrated that a radiomics signature is a potentially useful imaging biomarker for differentiating low from high DTD in patients with NSCLC based on contrast-enhanced computed tomography imaging [27]. With continuous advances in imaging technology and researches, some radiomics features are also applied to the prediction of pathologic stage in lung cancer. Tsutani et al. identified predictors of pathologic lymph node involvement in clinical stage IA lung adenocarcinoma [28]. In a clinico-pathologic study performed by Kaira et al., fluorine-18-alpha-methyltyrosine positron emission tomography was validated to be instrumental in diagnosis and staging of lung cancer [29]. Herein, we first conducted an equalization processing for imbalanced data sets, and then determined nine optimal image characteristics that may be related to the pathologic stages of LUAD and LUSC by means of Random Forest. Our 
prediction mode was validated internally and externally by means of multiple machine learning algorithms.

Radiomics applies machine learning algorithms to quantitative imaging data to characterize the tumour phenotype and predict clinical outcome. Recent breakthroughs in deep learning with applications in radiology, such as lung nodule malignancy classification, pathologic stage prediction and lymph node detection, have been instrumental in identifying disease-specific imaging biomarkers and improving the diagnostic performance [30-32]. For instance, Zhang et al. also unveiled optimal machine-learning algorithms for the radiomics-based prediction of local failure and distant failure in advanced nasopharyngeal carcinoma, which could enhance the applications of radiomics in precision oncology and clinical practice [33]. Furthermore, Ferreira et al developed and validated a radiomics signature that can be used for histopathological subtype diagnosis and metastatic prediction of lung cancer based on machine learning [34]. In this study, ROC curves and confusion matrix were employed for assessment on the classification accuracy of the prediction model, meanwhile, we also estimated the output quality of the classifier was also measured comprehensively using Precision-Recall metric. The prediction model that incorporated nine image features exhibited a high classification accuracy (all AUC >0.70), precision and recall scores $(\mathrm{AP}=0.60)$ in the training sets, while the predictive accuracy of the model in LUAD (AP $=0.84)$ was much higher than that in LUSC $(\mathrm{AP}=0.62)$ in the external validation. The results above also imply that there exists a large difference between LUAD and LUSC in terms of CT image features, and hence the two subtypes of NSCLC may be differentiated and predicted based on the difference of image features.

Nevertheless, it is noteworthy that there are some limitations in our radiomics analysis. Although our prediction model could be used for the precise tumor staging of lung cancer, some deviations may exist due to limited sample size. Moreover, the imbalanced data sets were subjected to equalization processing, while there are still some deficiencies, and thus a larger cohort would be needed for the further validation of the model. Furthermore, despite our focus is the staging of NSCLC, we still lack the CT images of healthy volunteers to be negative controls.

\section{Conclusions}

In conclusion, it is the first time that the significance of radiomics features in prediction of pathologic stages of NSCLC has been studied. Nine optimal image features were identified as predictive and diagnostic biomarkers for pathologic stages of NSCLC. Using multiple machine learning algorithms, our prediction model has been verified to effectively predict the tumor stages of NSCLC, especially for LUAD. Our findings not only extend the application of machine learning algorithms in CT image feature prediction for pathologic staging, but identify potential imaging biomarkers that can be used for diagnosis and prediction of pathologic stage in NSCLC.

\section{Additional files}

Additional file 1: TRIPOD Checklist: Prediction Model Development. (DOCX $88 \mathrm{~kb}$ )

Additional file 2: Table S2. Clinical information of NSCLC patients from TCIA database. (XLSX $15 \mathrm{~kb}$ )

Additional file 3: Table S3. Association between Radiomic Score and TNM factors. (XLSX $11 \mathrm{~kb}$ )

\section{Abbreviations}

AP: Average precision; AUC: Area under the curve; CT: Computed tomography; GLCM: Gray level co-occurrence matrix; IDN: Inverse difference normalized; LRLGLE: Long Run Low Gray Level Emphasis; LUAD: Lung adenocarcinoma; LUSC: Lung squamous cell carcinoma; NSCLC: Non-small cell lung cancer; OOB: Out of bag; RMS: Root mean squared; SAHGLE: Small Area High Gray Level Emphasis; TCIA: The cancer imaging archive

\section{Acknowledgements}

None.

\section{Funding}

The study was supported by Science and Technology Commission Shanghai Municipalit Project [Grant Number: No.18511102902]; Shanghai Hospital Development Center Project [Grant Number: SHDC22015033]; Shanghai Municipal Commission of Health and Family Planning Project [Grant Number: 20184Y0219]; and Interdisciplinary Program of Shanghai Jiao Tong University [Grant Number: YG2017QN661].

\section{Availability of data and materials}

All data generated or analyzed during this study are included in this published article. CT imaging data is publicly available in DICOM format at TCIA (https://wiki.cancerimagingarchive.net/display/Public/).

\section{Authors' contributions}

Contributing to the conception and design: LMY, GYT; Analyzing and interpreting data: LZ, GW; Drafting the article: LMY, ZML, GYT; Revising it critically for important intellectual content: JDY, QHC; Approving the final version to be published: all authors.

\section{Ethics approval and consent to participate}

Imaging, clinical characteristics and survival data have been de-identified by The Cancer Imaging Archive (TCIA) and are publicly available. Thus, no internal approval of an institutional review board was required for this study. Informed consent was collected by the TCIA Research Network.

\section{Consent for publication}

Not applicable.

\section{Competing interests}

The authors declare that they have no competing interests.

\section{Publisher's Note}

Springer Nature remains neutral with regard to jurisdictional claims in published maps and institutional affiliations.

\section{Author details}

'Department of Radiology, Shanghai Chest Hospital, The Affiliated Chest Hospital of Shanghai Jiaotong University, No. 241 Huaihai West Road, Xuhui District, Shanghai 200030, China. ${ }^{2}$ Department of Pathology, Shanghai Chest Hospital, The Affiliated Chest Hospital of Shanghai Jiaotong University, Shanghai 200030, China. ${ }^{3}$ Center for Statistics, Shanghai Chest Hospital, The 
Affiliated Chest Hospital of Shanghai Jiaotong University, Shanghai 200030, China. ${ }^{4}$ Center for Lung Tumor Clinical Medical, Shanghai Chest Hospital, The Affiliated Chest Hospital of Shanghai Jiaotong University, Shanghai 200030, China.

\section{Received: 19 August 2018 Accepted: 26 April 2019} Published online: 17 May 2019

\section{References}

1. Takenaka M, Hanagiri T, Shinohara S, Kuwata T, Chikaishi Y, Oka S, Shigematsu $Y$, Nagata $Y$, Shimokawa $H$, Nakagawa $M$, et al. The prognostic significance of HER2 overexpression in non-small cell lung cancer. Anticancer Res. 2011;31(12):4631-6.

2. de Castro J, Rodriguez MC, Martinez-Zorzano VS, Sanchez-Rodriguez P, Sanchez-Yague J. Erythrocyte fatty acids as potential biomarkers in the diagnosis of advanced lung adenocarcinoma, lung squamous cell carcinoma, and small cell lung cancer. Am J Clin Pathol. 2014;142(1):111-20.

3. Herbst RS, Morgensztern D, Boshoff $C$. The biology and management of non-small cell lung cancer. Nature. 2018;553(7689):446-54.

4. Hou S, Zhou S, Qin Z, Yang L, Han X, Yao S, Ji H. Evidence, mechanism, and clinical relevance of the Transdifferentiation from lung adenocarcinoma to squamous cell carcinoma. Am J Pathol. 2017;187(5):954-62.

5. Goke F, Perner S. Translational research and diagnostics in lung cancer. Pathologe. 2012:33(Suppl 2):269-72.

6. Valente IR, Cortez PC, Neto EC, Soares JM, de Albuquerque VH, Tavares JM. Automatic 3D pulmonary nodule detection in $C T$ images: a survey. Comput Methods Prog Biomed. 2016;124:91-107.

7. Thawani R, McLane M, Beig N, Ghose S, Prasanna P, Velcheti V, Madabhushi A. Radiomics and radiogenomics in lung cancer: a review for the clinician. Lung Cancer. 2018;115:34-41.

8. Leon-Atance P, Moreno-Mata N, Gonzalez-Aragoneses F, Canizares-Carretero MA, Garcia-Jimenez MD, Genoves-Crespo M, Honguero-Martinez AF Rombola CA, Simon-Adiego CM, Penalver-Pascual R. Multicenter analysis of survival and prognostic factors in pathologic stage I non-small-cell lung cancer according to the new 2009 TNM classification. Arch Bronconeumol. 2011;47(9):441-6.

9. Port JL, Kent MS, Korst RJ, Libby D, Pasmantier M, Altorki NK. Tumor size predicts survival within stage IA non-small cell lung cancer. Chest. 2003; 124(5):1828-33.

10. Nakayama H, Yamada K, Saito H, Oshita F, Ito H, Kameda Y, Noda K. Sublobar resection for patients with peripheral small adenocarcinomas of the lung: surgical outcome is associated with features on computed tomographic imaging. Ann Thorac Surg. 2007;84(5):1675-9.

11. Yu W, Tang C, Hobbs BP, Li X, Koay EJ, Wistuba II, Sepesi B, Behrens C, Rodriguez Canales J, Parra Cuentas ER, et al. Development and validation of a predictive Radiomics model for clinical outcomes in stage I non-small cell lung Cancer. Int J Radiat Oncol Biol Phys. 2017.

12. van Timmeren JE, Leijenaar RTH, van Elmpt W, Reymen B, Oberije C, Monshouwer R, Bussink J, Brink C, Hansen O, Lambin P. Survival prediction of non-small cell lung cancer patients using radiomics analyses of conebeam CT images. Radiother Oncol. 2017;123(3):363-9.

13. Shim SS, Lee KS, Kim BT, Chung MJ, Lee EJ, Han J, Choi JY, Kwon OJ, Shim YM, Kim S. Non-small cell lung cancer: prospective comparison of integrated FDG PET/CT and CT alone for preoperative staging. Radiology. 2005;236(3):1011-9.

14. Choi ER, Lee HY, Jeong JY, Choi YL, Kim J, Bae J, Lee KS, Shim YM. Quantitative image variables reflect the intratumoral pathologic heterogeneity of lung adenocarcinoma. Oncotarget. 2016;7(41):67302-13.

15. Lambin P, van Stiphout RG, Starmans MH, Rios-Velazquez E, Nalbantov G, Aerts HJ, Roelofs E, van Elmpt W, Boutros PC, Granone P, et al. Predicting outcomes in radiation oncology--multifactorial decision support systems. Nat Rev Clin Oncol. 2013;10(1):27-40.

16. Lambin P, Rios-Velazquez E, Leijenaar $R$, Carvalho $S$, van Stiphout RG, Granton P, Zegers CM, Gillies R, Boellard R, Dekker A, et al. Radiomics: extracting more information from medical images using advanced feature analysis. Eur J Cancer. 2012;48(4):441-6.

17. Soufi M, Arimura H, Nakamoto $T$, Hirose TA, Ohga S, Umezu Y, Honda $H$, Sasaki T. Exploration of temporal stability and prognostic power of radiomic features based on electronic portal imaging device images. Phys Med. 2018;46:32-44.
18. Meng Y, Zhang Y, Dong D, Li C, Liang X, Zhang C, Wan L, Zhao X, Xu K,

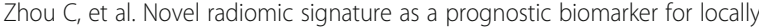
advanced rectal cancer. J Magn Reson Imaging. 2018.

19. Li Q, Kim J, Balagurunathan Y, Liu Y, Latifi K, Stringfield O, Garcia A, Moros EG, Dilling $T J$, Schabath MB, et al. Imaging features from pretreatment $C T$ scans are associated with clinical outcomes in nonsmall-cell lung cancer patients treated with stereotactic body radiotherapy. Med Phys. 2017:44(8):4341-9.

20. Kim H, Park CM, Keam B, Park SJ, Kim M, Kim TM, Kim DW, Heo DS, Goo JM. The prognostic value of $C T$ radiomic features for patients with pulmonary adenocarcinoma treated with EGFR tyrosine kinase inhibitors. PLoS One. 2017;12(11):e0187500.

21. Ravanelli M, Farina D, Morassi M, Roca E, Cavalleri G, Tassi G, Maroldi R. Texture analysis of advanced non-small cell lung cancer (NSCLC) on contrast-enhanced computed tomography: prediction of the response to the first-line chemotherapy. Eur Radiol. 2013;23(12):3450-5.

22. Fernandez-Delgado M, Sirsat MS, Cernadas E, Alawadi S, Barro S, FebreroBande M. An extensive experimental survey of regression methods. Neural Netw. 2018;111:11-34.

23. Hu Y, Lu Y, Wang S, Zhang M, Qu X, Niu B. Application of machine learning approaches for the design and study of anticancer drugs. Curr Drug Targets. 2018.

24. Fave X, Zhang L, Yang J, Mackin D, Balter P, Gomez D, Followill D, Jones AK, Stingo F, Liao Z, et al. Delta-radiomics features for the prediction of patient outcomes in non-small cell lung cancer. Sci Rep. 2017;7(1):588.

25. Coroller TP, Grossmann P, Hou Y, Rios Velazquez E, Leijenaar RT, Hermann G, Lambin P, Haibe-Kains B, Mak RH, Aerts HJ. CT-based radiomic signature predicts distant metastasis in lung adenocarcinoma. Radiother Oncol. 2015; 114(3):345-50.

26. Ko KH, Hsu HH, Huang TW, Gao HW, Cheng CY, Hsu YC, Chang WC, Chu CM, Chen JH, Lee SC. Predictive value of 18F-FDG PET and CT morphologic features for recurrence in pathological stage IA non-small cell lung cancer. Medicine (Baltimore). 2015;94(3):e434.

27. Guo C, Wang X, Chen LP, Li M, Li M, Hu YH, Ding WH, Wang X. Long noncoding RNA MALAT1 regulates ovarian cancer cell proliferation, migration and apoptosis through Wnt/beta-catenin signaling pathway. Eur Rev Med Pharmacol Sci. 2018;22(12):3703-12.

28. Tsutani $Y$, Miyata $Y$, Nakayama H, Okumura S, Adachi S, Yoshimura M, Okada M. Prediction of pathologic node-negative clinical stage IA lung adenocarcinoma for optimal candidates undergoing sublobar resection. J Thorac Cardiovasc Surg. 2012;144(6):1365-71.

29. Kaira K, Oriuchi N, Otani Y, Shimizu K, Tanaka S, Imai H, Yanagitani N, Sunaga N, Hisada T, Ishizuka T, et al. Fluorine-18-alpha-methyltyrosine positron emission tomography for diagnosis and staging of lung cancer: a clinicopathologic study. Clin Cancer Res. 2007;13(21):6369-78.

30. Tu SJ, Wang CW, Pan KT, Wu YC, Wu CT. Localized thin-section CT with radiomics feature extraction and machine learning to classify earlydetected pulmonary nodules from lung cancer screening. Phys Med Biol. 2018;63(6):065005.

31. Kim JK, Yook IH, Choi MJ, Lee JS, Park YH, Lee JY, Choi IY. A performance comparison on the machine learning classifiers in predictive pathology staging of prostate Cancer. Stud Health Technol Inform. 2017;245:1273.

32. Ehteshami Bejnordi B, Veta M, Johannes van Diest P, van Ginneken B, Karssemeijer N, Litjens G, van der Laak J, The CC, Hermsen M, Manson QF, et al. Diagnostic assessment of deep learning algorithms for detection of lymph node metastases in women with breast Cancer. JAMA. 2017;318(22): 2199-210.

33. Zhang B, He X, Ouyang F, Gu D, Dong Y, Zhang L, Mo X, Huang W, Tian J, Zhang $S$. Radiomic machine-learning classifiers for prognostic biomarkers of advanced nasopharyngeal carcinoma. Cancer Lett. 2017:403:21-7.

34. Ferreira Junior JR, Koenigkam-Santos M, Cipriano FEG, Fabro AT, Azevedo-Marques PM. Radiomics-based features for pattern recognition of lung cancer histopathology and metastases. Comput Methods Prog Biomed. 2018;159:23-30. 